Bond University

Research Repository

\title{
Effects of acute alcohol consumption on executive cognitive functioning in naturalistic settings
}

Lyvers, Michael; Tobias-Webb, Juliette

Published in:

Addictive Behaviors

DOI:

10.1016/j.addbeh.2010.06.022

Licence:

CC BY-NC-ND

Link to output in Bond University research repository.

Recommended citation(APA):

Lyvers, M., \& Tobias-Webb, J. (2010). Effects of acute alcohol consumption on executive cognitive functioning in naturalistic settings. Addictive Behaviors, 35(11), 1021-1028. https://doi.org/10.1016/j.addbeh.2010.06.022

\footnotetext{
General rights

Copyright and moral rights for the publications made accessible in the public portal are retained by the authors and/or other copyright owners and it is a condition of accessing publications that users recognise and abide by the legal requirements associated with these rights.

For more information, or if you believe that this document breaches copyright, please contact the Bond University research repository coordinator.
} 
Effects of Acute Alcohol Consumption on Executive Cognitive Functioning in Naturalistic Settings

Michael Lyvers, Ph.D.

Juliette Tobias-Webb, Honours

Department of Psychology

Bond University

Gold Coast Q1d 4229

Australia

Corresponding author: Michael Lyvers

Phone: (61) (75) 5952565

Fax: (61) (75) 5952540

Email: mlyvers@bond.edu.au 


\begin{abstract}
Laboratory studies have demonstrated that acute alcohol intoxication can disrupt performance on neuropsychological tests of executive cognitive functioning such as the Wisconsin Card Sorting Test (WCST). However, the generalizability of such findings to typical self-regulated alcohol intake in social settings can be questioned. In the present study, 86 young adults were recruited at Australian bars to perform a computer version of the WCST. Participants displayed blood alcohol concentrations (BACs) across a range from 0 to $0.15 \%$. Although self-report measures of typical alcohol consumption, impulsivity, and frontal lobe related everyday functioning were all intercorrelated in line with other recent findings, multiple regression indicated that these measures did not predict perseverative errors (PE) nor non-perseverative errors (NPE) on the WCST, whereas BAC uniquely predicted PE but not NPE. The results were consistent with a dosedependent selective disruption of prefrontal cortical functioning by alcohol. There were no differences in performance between participants tested on the ascending limb of the BAC curve and those tested on the descending limb. Alcohol-associated perseveration may reflect the inhibitory effect of alcohol-induced dopamine release in the prefrontal cortex.
\end{abstract}

KEYWORDS: alcohol, executive functioning, prefrontal cortex 
The Effects of Acute Alcohol Consumption on Executive Cognitive Functioning in Naturalistic Settings

A variety of neurophysiological and neuropsychological evidence indicates that the prefrontal cortices are disproportionately affected by both acute and chronic alcohol consumption (Lyvers, 2000, Oscar-Berman \& Marinkovic, 2007). The prefrontal cortex is the primary neurological substrate mediating executive cognitive functions, an umbrella term that subsumes problem solving, cognitive flexibility, planning, organization, abstract reasoning and social conduct (Giancola, 2007). As executive cognitive functioning is concerned with the ongoing regulation of behavior and the anticipation of future consequences of actions, its temporary disruption by alcohol can increase the likelihood that intoxicated individuals will engage in risky, impulsive behaviors such as unprotected sex (Davis et al., 2009; Lyvers, Cholakians, Puorro \& Sundram, 2010a), violence (Giancola \& Zeichner, 2007) and drunk driving (Finnigan \& Hammersley, 1992). Understanding the nature and breadth of alcohol's acute effects on executive cognitive functioning is thus imperative.

Using a balanced placebo design, Peterson, Rothfleisch, Zelazo and Pihl (1990) reported that participants given high doses of alcohol (inducing a mean blood alcohol concentration/BAC of $0.09 \%)$ exhibited pronounced deficits as compared to those given low doses of alcohol $(M=$ $0.02 \% \mathrm{BAC}$ ) on four out of six neuropsychological indices of prefrontal cortical functioning and on four out of six neuropsychological indices of temporal lobe functioning. At moderate doses $(M=0.06 \% \mathrm{BAC})$, two of the prefrontal measures showed impairment, but no impairment was found for any of the temporal lobe measures. Their findings suggested that prefrontal cortical functioning may be susceptible to dose-dependent disruption by alcohol, such that increasing BAC exerts a progressive and selective influence, until at high BAC more widespread regions of cortex are affected nonselectively. Subsequent studies have consistently reported selective alcohol-induced impairments on various neuropsychological indices of prefrontal cortical 
functioning (Lyvers \& Maltzman, 1991; Peterson, Finn \& Pihl, 1992) with no apparent effect on “nonfrontal” tasks (Hoaken, Assaad \& Pihl, 1998; Weissenborn \& Duka, 2003) at moderate BACs. For example, Lyvers and Maltzman administered the Wisconsin Card Sorting Test (WCST) - the so-called "gold standard" among neuropsychological tests of prefrontal cortical functioning (Podell, Lovell, Zimmerman \& Goldberg, 1995) - to social drinkers who had been given a moderate dose of alcohol (inducing peak BACs of .05\%) or placebo in a balanced placebo design. Lyvers and Maltzman implemented a dual run WCST administration procedure previously shown to render the test more specifically sensitive to the effects of prefrontal cortex damage than the standard procedure (Stuss et al., 1983) and which more selectively activates the prefrontal cortex than the standard procedure according to brain imaging studies (Berman et al., 1995; Smith et al., 1997). Alcohol selectively increased perseverative errors (PE) but not nonperseverative errors (NPE). PE refers to errors due to persisting with a previously reinforced but currently inappropriate response; on the WCST, PE tend to be selectively elevated in patients with localized prefrontal cortex damage but not in patients with damage localized to temporal, parietal or occipital lobes (Mountain \& Snow, 1993; Stuss et al., 2000; Strauss, Sherman \& Spreen, 2006). Selective increase in PE was thus interpreted by Lyvers and Maltzman as reflecting a relatively selective depressant action of alcohol on the prefrontal cortex, consistent with neurophysiological evidence (e.g., de Wit, Metz, Wagner \& Cooper, 1990; Volkow et al., 1994). Although all participants had said they fully expected alcohol to impair their problemsolving ability, alcohol expectancy had no effect on WCST performance in Lyvers and Maltzman's study and also had no effect on problem-solving in the study by Peterson et al. (1990). The absence of expectancy effects was consistent with the notion that alcohol expectancy affects social behaviors but not cognitive performance (Hull \& Bond, 1986), hence control of alcohol expectancy via a balanced placebo design does not appear to be necessary in neuropsychological investigations of alcohol effects.

More recently, Weissenborn and Duka (2003) found that a moderate dose of alcohol 
(inducing a mean BAC of .06\%) selectively impaired performance measures that were specifically related to prefrontal cortical functioning while sparing those related to temporal lobe functioning. They concluded that their findings supported those of Lyvers and Maltzman (1991). Although laboratory studies such as these and the earlier study by Peterson et al. (1990) all support the notion that acute alcohol consumption selectively affects neuropsychological indices of prefrontal cortical functioning, this hypothesis has not been tested in naturalistic settings, hence these findings lack ecological validity. The latter is important to establish because without it, one can only speculate as to the relevance of behavior in laboratory studies (Finnigan \& Hammersley, 1992). When individuals consume alcohol in naturalistic environments such as bars, alcohol ingestion is generally under the control of the drinker as well as being influenced by the convivial social environment. In laboratory settings, individuals are exposed to a very different, far more regimented social environment in which they cannot monitor or control their intake of alcohol and cannot achieve and maintain a desired level of intoxication as they are required to drink a certain amount of alcohol (or placebo) in a certain period of time. The generalizability of research conducted entirely in laboratory settings is thus limited with regard to the effect of acute alcohol consumption on executive cognitive functioning, hence such findings need to be validated under naturalistic conditions. For example, in naturalistic settings social drinkers may commonly titrate their alcohol intake over time in order to maintain a relaxed and sociable state and/or avoid obvious impairment. Both the rate and duration of alcohol intake have been found to influence behavior beyond simple consideration of BAC alone (Fleming et al., 2001). Moreover, due to ethical constraints the doses of alcohol administered in laboratory studies may not be sufficient to reveal the full impact of acute alcohol intoxication on behavior. The naturalistic drinking setting of an Australian bar potentially provides not only a sample of drinkers in a typical drinking context, but also includes drinkers who have achieved a wide range of $\mathrm{BAC}$ that at the upper end is likely to exceed the levels allowed by most ethics committees for experimenter-administered alcohol. The present study recruited patrons of 
popular Australian bars across a wide range of BAC ( 0 to .15\%) and tested them on the dual run WCST to assess alcohol's dose-dependent effect on this "gold standard" test of prefrontal cortical executive cognitive functioning, with the aim of determining whether the earlier laboratory results obtained by Lyvers and Maltzman (1991) are generalizable to naturalistic drinking contexts.

An issue which laboratory studies have largely ignored when considering alcohol effects on executive cognitive functioning is the possible differential effect of alcohol across the biphasic BAC curve. Alcohol is often said to produce a stimulant-like effect on the ascending limb of the BAC curve followed by an undesirable sedative effect on the descending limb (Pihl, Paylan, Gentes-Hawn \& Hoaken, 2003). A few studies have indicated that at moderate doses some types of impairments may be restricted to the ascending limb of the BAC curve, including disruptions in motor functioning (Holdstock \& de Wit, 1998), attention (Hurst \& Bagley, 1972) and immediate memory (Jones, 1973). Increased sexual arousal (Davis et al., 2009), unprotected sexual intentions (Davis et al.) and increased aggression (Giancola \& Zeichner, 1997) also may be more prevalent on the ascending limb than the descending limb. To examine BAC limb differences in alcohol's effect on executive cognitive functioning. Pihl et al. (2003) tested intoxicated participants on executive tasks at either $0.08 \% \mathrm{BAC}$ on the ascending limb or $0.08 \%$ BAC on the descending limb. Consistent with other research (Lyvers \& Maltzman, 1991; Peterson et al., 1990; Weissenborn \& Duka, 2003), Pihl et al. found that participants who had consumed alcohol showed impairments on two executive cognitive measures when compared to the placebo control $(0 \% \mathrm{BAC})$ condition, however there was greater impairment among those on the descending BAC limb compared to those on the ascending limb on one of the measures. The present study examined possible BAC limb effects on executive cognitive performance as measured by the WCST by taking into account the BAC limb (ascending or descending) that intoxicated participants were on during testing.

The primary aim of the present study was to assess the generalizablity of previous 
laboratory work by investigating the effects of acute alcohol consumption on executive cognitive performance in the naturalistic settings of three popular Australia bars. Volunteers were recruited on site and were tested using the dual run WCST. Additionally, to control for the possible influence of individual difference factors that have been linked to executive cognitive functioning, three well-established questionnaires were administered: the Alcohol Use Disorders Identification Test (AUDIT), the Barratt Impulsiveness Scale 11 (BIS-11), and the Frontal Systems Behavior Scale (FrSBe). The AUDIT assesses differences in individual drinking patterns and alcoholrelated harm (Babor, de la Fuente, Saunders \& Grant, 1992), and was used to control for potential prefrontal cortical dysfunction associated with chronic heavy drinking (Giancola, 2007; Lyvers, 2000; Oscar-Berman \& Marinkovic, 2007; Ratti, Bo, Giardini \& Soragna, 2002). The BIS-11 is one of the most widely used and validated measures of trait impulsivity (Patton, Stanford \& Barratt, 1995), which is well-known to be associated with increased risk for early onset alcoholism (Pardo, Aguilar, Molinuevo \& Torrubia, 2007). As impulsivity is widely regarded as a "frontal" personality trait (Chen et al., 2007), an individual's executive cognitive performance may, in part, reflect their genetically influenced impulsive behavior, hence use of the BIS-11 allowed individual differences in impulsivity to be controlled and examined separately. The FrSBe assesses self-reported everyday functioning in behavioral domains related to the functional integrity of the prefrontal cortices (Grace \& Malloy, 2001), and was used to control for possible trait differences in prefrontal cortical functioning. Based on the previous work cited above, after taking into account the potential contributions of AUDIT, BIS-11 and FrSBe scores, BAC was expected to uniquely predict PE but not NPE on the second run of the WCST, consistent with the hypothesized selective action of alcohol on prefrontal cortex. Given the inconsistent findings previously reported, no specific predictions were made concerning possible differential BAC limb effects on executive cognitive performance in the present study.

Method

\section{Participants}


Out of 98 individuals tested, the 86 participants who provided useable data included university students $(n=56 ; 65 \%)$ and non-student members of the local community $(n=29$; $34 \%)$ aged $18-30$ years $(M=22.14, S D=3.22)$ who were recruited from three bars across the Southeastern Queensland region. There were 48 (56\%) males and 38 (44\%) females. Prospective participants were excluded from the study if they were not native English speakers, not between 18 and 30 years of age, reported having a learning disability or a history of psychological or neurological illness, reported the use of any illicit drug within the previous 48 hours, if their BAC exceeded $0.15 \%$, or if they failed to comprehend the WCST task instructions as evidenced by extreme outlying error scores (identified by Boxplots; 12 of the original 98 tested were excluded on this basis as they failed to complete more than a single category even on the second run). Individuals displaying $\mathrm{BAC}$ of over $0.15 \%$ were likely to be drastically impaired in terms of their ability to give informed consent and hence were excluded on ethical grounds as per the requirements of the Bond University Human Research Ethics Committee (BUHREC). No incentives were offered to participants unless they were undergraduate psychology students eligible to receive one credit point for their participation $(n=6)$.

\section{Materials}

The Alcohol Use Disorders Identification Test (AUDIT; Babor et al., 1992) is a 10 -item self-report measure widely used in clinical and community settings when screening the adult population for risky drinking patterns. Questions 1 to 3 assess level of alcohol consumption (e.g., 'how often do you have a drink containing alcohol?'). Questions 4 to 6 relate to alcohol dependence (e.g., 'how often during the last year have you found that you were not able to stop drinking once you had started?'). Questions 7 to 10 address alcohol-associated problems (e.g., 'how often during the last year have you had a feeling of guilt or remorse after drinking?'). AUDIT items are scored from 0 to 4 . A participant's total AUDIT score is simply the sum of their responses to the 10 items, yielding possible totals ranging from 0 to 40 . A score of 0 to 7 is indicative of low risk alcohol consumption. A score of 8 to 15 indicates risky or hazardous alcohol consumption, whereas 
a score of 16 or higher suggests the respondent is drinking at a harmful level. The test takes approximately two minutes to administer and no items are reversed scored. The AUDIT has been shown to have excellent internal consistency with a median reliability coefficient of 0.83 (range $=$ 0.75 - 0.97) for studies published between 2002 and 2006 using clinical and non-clinical populations (Reinert \& Allen, 2007). The measure has good to excellent temporal stability with testretest reliabilities for general population samples ranging from $r=.87$ over a one week time period (Rubin et al., 2006) to $r=0.93$ and 0.95 over a one month time period (Bergman \& Källmén, 2002; Dybek et al., 2006). Adequate criterion validity has also been established with sensitivity for alcohol misuse diagnoses across primary care samples ranging from 0.60 to 0.95 and specificity ranging from 0.84 to 0.96 at the cut off score of 8 (Berner, Kriston, Bentele \& Harter, 2007). Concurrent validity has been established using the Michigan Alcoholism Screen Test (Bohn, Babor \& Kranzler, 1995; Pal, Jena, \& Yadav, 2004).

The Participant Information Battery is a 12-item self-report inventory designed for this study. It contains questions regarding the participant's age, gender, first language, completed years of education, current occupational status, marital status, the history and presence of a psychological or neurological illness, current learning disabilities, use of illicit drugs other than alcohol within the last 48 hours and the quantity and frequency of tobacco and illicit drug use. Responses on this questionnaire were used for screening purposes and also yielded demographic characteristics of the sample.

The Alcolizer HH-1 (Alcolizer Technology, 2007), an Australian certified breathalyzer, was used to measure BAC. This breathalyzer provides accurate BAC readings to one hundredth of one percent between temperatures of 5 to 50 degrees Celsius. The HH-1 informs the user when recalibration is required (i.e., after 300 breath samples or six months, whichever comes first). The device was recalibrated by the manufacturer prior to data collection and recalibrated again after two months of use in this study.

The Barratt Impulsiveness Scale Version 11 (BIS-11; Patton et al., 1995) is a 30-item 
self-rating inventory designed to assess trait impulsivity in clinical and non-clinical adult populations. Items assess non-planning impulsivity, an inability to plan for events (8 items; e.g., 'I plan trips well ahead of time,' reversed scored); motor impulsivity, or acting in a spontaneous, erratic manner ( 8 items; e.g., 'I act on the spur of the moment'); and attentional impulsivity (11 items; e.g., 'I can only think about one problem at a time'). All items are rated on a four-point Likert scale ranging from 1 ('rarely/never') to 5 ('almost always/always') and 11 items are reverse scored. Items are summed together to create a total scale score ranging from 30 to 150 reflecting level of impulsivity. The test takes approximately five minutes to administer. The BIS-11 has been shown to have good internal consistency with reliability coefficients ranging from 0.79 to 0.83 for undergraduates, community members, substanceabusers, general psychiatric patients and prison inmates (Barratt \& Patton, 1983; Patton et al., 1995; Spinella, 2007). It has also been shown to have good temporal stability with a test-retest reliability coefficient of $r=0.60$ over one year (Luengo, Carrillo-de-la-Pena \& Otero, 1991). Positive correlations with established neuropsychological measures such as a Go/No-Go test and an Antisaccade test support the convergent validity of the BIS-11 (Spinella, 2004).

The Frontal Systems Behavior Scale (FrSBe; Grace \& Malloy, 2001) is a 46 item selfreport inventory designed to identify and quantify behavioral disturbances associated with frontal lobe damage in individuals aged between 18 and 95. Factor analytic studies using several different neurological populations (Stout, Ready, Grace, Malloy \& Paulsen, 2003) have confirmed that the scale yields a total composite score indicating the overall presence of frontal lobe related behavior problems and three subscale scores measuring Apathy (poor initiation and reduced interest; 14 items, e.g., 'Am inflexible, unable to change routines'), Disinhibition (distractibility, impulsivity and social disinhibition; 15 items, e.g., 'I do things impulsively') and Executive Dysfunction (impairment in motor planning, mental flexibility, working memory and conceptual reasoning; 17 items, e.g., 'Mix up a sequence, get confused when doing several things in a row'). These three subscales were designed to detect behavioral syndromes associated with 
damage to the anterior cingulate, orbital frontal and dorsolateral frontal systems, respectively.

On the original questionnaire, brain-injured patients are required to fill in two adjacent boxes, one asking about pre-injury behavior and one about present (post-injury) behavior. In the present study, participants were only asked to provide present ratings of themselves.

All FrSBe items are rated on a five-point Likert scale with responses ranging from 1 ('almost never') to 5 ('almost always'). The first 32 items represent deficits and are rated accordingly, whereas the final 14 items reflect executive functioning and the Likert descriptors are reversed (e.g., 'Use strategies to remember important things,' where 1 = 'almost always' and $5=$ 'almost never'). All items are summed together to create a total score ranging from 46 to 230. Higher ratings indicate greater degrees of abnormal "frontal lobe" behavior. The FrSBe has been shown to have excellent internal consistency $(r=0.96)$, split half reliability $(0.93)$ and temporal stability ( $r=0.78$ over a three month time period) for normal and clinical samples (Grace, Stout \& Malloy, 1999; Velligan, Ritch, Sui, Di Cocco \& Huntzinger, 2002). The sensitivity of the scale to prefrontal cortical dysfunction has been demonstrated in a variety of neurological, psychiatric and non-clinical populations (Chiaravalloti \& DeLuca, 2003; Spinella, 2004; Stout et al.; Velligan et al.). Convergent validity is supported as the FrSBe has been found to correlate with objective measures of executive dysfunction (Norton, Malloy \& Salloway, 2001).

The Wisconsin Card Sorting Test -64 (Kongs, Thompson, Iverson \& Heaton, 2000) is the abbreviated form of the standard Wisconsin Card Sorting Test (WCST; Heaton, 1981), and is designed to assess executive cognitive functioning (i.e., cognitive flexibility, abstract reasoning skills and planning). For ease of administration and to minimize procedural and scoring errors, the computerized version of the test was administered using the Psychological Experiment Building Language (PEBL) test battery version 0.09 (Mueller, 2008). Tien et al. (1996) reported that there was minimal, if any, difference on PE and NPE measures between the original card version and the computerized version of the WCST. In the latter, each participant is presented with four evenly spaced stimulus cards on the top part of a computer screen; the first card has a red triangle, 
the second has two green stars, the third has three yellow crosses and the fourth has four blue circles. Sixty-four response cards, which also vary in geometric form, color and number, are then presented individually in the bottom right hand corner of the screen, and the participant's task is to match each response card to one of the four stimulus cards according to a principle that the participant must deduce from the feedback provided. Each response card is presented on the screen until the participant responds with a button press that corresponds to one of the stimulus cards (i.e., $1=$ one red triangle, $2=$ two green stars, $3=$ three yellow crosses, $4=$ four blue circles). In the present study, each of the four numbered buttons was colored the same as its corresponding stimulus card. Although on the first WCST run the participant is not instructed on how to match the cards, the computer program provides feedback, displaying the word 'correct' or 'incorrect' each time a sorting choice is made. The participant is required to sort by one principle first (e.g., color) and once 10 consecutive correct responses have been achieved, the required sorting principle shifts, without warning, to another sorting principle (e.g., form or number). The order of categories is standardized following the order of color, form, number, color, form, number. This procedure continues until the participant has either successfully completed six sorting categories or until all 64 cards have been sorted. Successful performance of the WCST requires the participant to utilize the feedback provided by the program and continuously identify and act in accordance to the correct sorting rule. The test takes approximately 10 minutes to administer. For the purposes of the present study, PE and NPE were the primary measures of interest (as described previously above), and the number of categories completed (CC) was also examined as it is a standard measure of performance. PE refers to the number of times the participant persisted in making an incorrect sorting choice that was previously correct. NPE refers to incorrect responses other than PE, e.g., prematurely switching set.

\section{Procedure}

Once informed consent was obtained from bar managers, prospective participants were 
approached at three bars between $7 \mathrm{pm}$ and $10 \mathrm{pm}$ on various nights over a three month period. After introducing the study and outlining the exclusion criteria, prospective participants were asked if they would like to take part in the study. Volunteers were asked not to eat or drink any more until after they completed their participation. Each participant was then individually directed to the testing area, which was a relatively quiet area containing a table and two chairs, and which minimized potential distractions such as crowds and sources of loud noise or music. Each participant was provided with an explanatory statement describing the purpose and procedures of the study, and was asked to sign a liability disclaimer stating that the researchers and their affiliated university were not liable for the participant's actions after the testing session was completed. The participant was then asked to rinse their mouth out with water using disposable cups provided by the researcher; this was to eliminate residual alcohol in the mouth which might otherwise inflate BAC readings. Each participant then completed the Participant Information Battery, BIS-11, AUDIT and FrSBe, after which they were instructed to rinse their mouth out with water again and blow into the mouthpiece of the Alcolizer $H H-1$ for approximately five seconds until the researcher said to stop. The time required to fill out the questionnaires (approximately 15 minutes) was sufficient to obtain accurate BAC readings (Bailey, 2005). BAC was recorded on a sheet of paper that was kept out of the sight of the participant. The WCST-64 was then administered on the color monitor of a Hewlett-Packard Pavilion computer with a 17 inch screen. For the first run, the standard instructions were read aloud to the participant. The participant was told to match each response card to one of the four stimulus cards by pressing the corresponding button on the response panel. The experimenter did not describe the sorting principles or the nature of the shift from one sorting category to the next. The participant's task was thus to match each response card to one of the stimulus cards according to a principle that must be deduced from the pattern of 'correct' or 'incorrect' responses provided by the computer program. The test continued until each participant completed six sorting categories or until the 64 response cards were used, whichever came first. 
Following the completion of the first run, a second BAC reading was taken following the same procedure as before. The participant was then required to complete the WCST for a second time with the following instruction: "There are three ways to match the cards, by color, shape or number; please try the test again." Following the completion of the two WCST runs, each participant was breathalyzed for a third and final time. The participants were asked not to tell anyone how to play the "game" (WCST) as this would disrupt the research findings. Each participant was also instructed that if drinking they should not drive. They were then provided with their three BAC readings, warned against using these readings as a guide for making decisions and thanked for their time. Each test session took approximately 30 minutes in total duration, with BAC readings taken at roughly 10 minute intervals (i.e., immediately before first run, immediately after first run, and immediately after second run).

\section{Results}

All analyses were conducted using the Statistical Package for the Social Sciences (SPSS) version 17.0. No severe violations of skew or kurtosis were detected for the predictor variables, however, as expected a positive skew was noted for WCST second run PE and NPE scores. The multivariate assumptions of linearity, singularity, multicollinearity and independence of errors were found to be satisfactory for all variables. Independent samples t-tests revealed that the predictor and criterion variables did not significantly differ between the community sample and the university sample, which justified running the subsequent analyses with the samples combined. The overall sample was examined for possible gender differences on all measures (including the primary WCST outcome measures, second run PE and NPE, as well as BAC) with no overall multivariate effect according to Pillai's Trace, $F(6,79)=1.22, p=.30$; however significant univariate gender differences were found for AUDIT scores, $F(1,84)=$ $6.17, p=.015$, and FrSBe scores, $F(1,84)=4.67, p=.033$. Men scored higher on AUDIT $(M$ $=15.50, S D=7.77)$ than women $(M=11.47, S D=7.07)$, and men scored higher on $\operatorname{FrSBe}(M$ $=99.88, S D=19.19)$ than women $(M=90.84, S D=19.31)$. These gender differences were 
entirely consistent with previous work (AIHW, 2008; Bohn et al., 1995; Chiaravalloti \& DeLuca, 2003; Lyvers, Czerczyk, Follent \& Lodge, 2009; Velligan et al., 2002).

Table 1 presents the overall means and standard deviations for the quantitative variables of interest (note that BAC refers here and below to the average of the three measurements taken for each participant across the testing period). Those with BACs greater than zero showed a range of $.01 \%$ to $.15 \%$. Scores on both the BIS-11 and the FrSBe were similar to those reported in normative studies (Grace \& Malloy, 2001; Spinella, 2007). However, on the AUDIT, $23.3 \%$ of the participants reported Low Risk drinking $(n=20), 43 \%$ were defined as Hazardous drinkers $(n=37)$ and $33.7 \%$ of participants scored within the Harmful range $(n=$ 29) according to AUDIT scoring criteria. These results indicated higher alcohol consumption than the Australian Institute of Health and Welfare (AIHW, 2008) national averages, which was anticipated given that the present sample was recruited solely at bars.

\section{Correlations}

Intercorrelations among variables of primary interest are presented in Table 2. The correlation coefficients indicated that, consistent with expectations, BAC showed a moderate positive association with WCST second run PE (as illustrated in Figure 1) yet was not significantly correlated with WCST second run NPE, a pattern consistent with selective disruption of prefrontal cortical functioning by alcohol. BAC was also significantly negatively correlated with Categories Completed (CC). Other significant associations of interest included the moderate positive correlations among BIS-11, AUDIT and FrSBe scores, consistent with other recent work (Lyvers, Duff \& Hasking, 2010). BAC was significantly positively correlated with BIS-11, AUDIT, and FrSBe, indicating that higher levels of self-reported impulsivity, typical alcohol consumption, and everyday frontal lobe related behavior problems were all associated with greater alcohol intake prior to testing. 
A hierarchical multiple regression was performed with the BIS-11, AUDIT, FrSBe (Step 1) and BAC (Step 2) as the predictors, and WCST second run PE (the operational index of prefrontal cortical dysfunction) as the criterion. Table 3 shows the unstandardised (B) and standardised $(\beta)$ coefficients for each predictor at each step. As can be seen in Table 3, at Step 1 with the BIS-11, AUDIT and FrSBe in the model, R for Regression was not significant, $F(3,82)=.77, p$ $=.51$, with $<3 \%$ of the variance in PE accounted for by the predictors. The addition of BAC at Step 2 produced a significant increment in $\mathrm{R}$, with an additional $10.5 \%$ of the variance in PE explained by that variable, Fchange $(1,81)=9.78, p=.002$. Together all four variables significantly predicted PE scores, $\mathrm{R}=.36, F(4,81)=3.08, p=.02$. At Step 2 , only BAC contributed uniquely to the prediction of PE, $\beta=.35, t(84)=3.13, p=.002$. The BIS-11, $\beta=.091, t<1$, AUDIT, $\beta=.003, t<$ 1 , and FrSBe, $\beta=-.146, t(84)=-1.11, p=.27$, were not significant.

A second hierarchical multiple regression was performed using an equivalent model to that described above, with the exception that WCST second run NPE (the control index of general brain dysfunction) was the criterion. Table 4 shows the unstandardised (B) and standardised $(\beta)$ coefficients for each predictor at each step. As can be seen in Table 4, with the BIS-11, AUDIT, and FrSBe in the model at Step 1, $\mathrm{R}$ was not significant, $F(3,82)=0.48, p=$ .70 ; less than $2 \%$ of the variance in NPE could be explained by the predictors. The addition of BAC at Step 2 did not significantly add to the prediction of NPE, $\operatorname{Fchange}(1,81)=3.04, p=$ .09 , and together all four predictors accounted for $5.3 \%$ of the variance in the criterion, $F(4$, $85)=1.13, p=.35$, n.s.. At Step 2 , none of the variables contributed uniquely to the prediction of NPE, all $t<1.74$, n.s.

\section{First WCST Run Correlations and Regressions}

As first run WCST performance was expected to be less sensitive to prefrontal cortex dysfunction than second run WCST performance, no specific predictions were made; however, exploratory analyses revealed that significant correlations with BAC were nevertheless obtained for first run CC, $r=-.39, p<.01$, and PE, $r=.23, p<.05$. These results are of course 
consistent with the hypothesis that alcohol selectively disrupts prefrontal cortical functioning. The regression model described above did not reach significance for first run $\mathrm{PE}, F(4,81)=$ $2.18, p=.08$, however for CC the model was significant with the addition of BAC at Step 2, $F(4,81)=4.31, p=.003$, with $17.5 \%$ of the variance in CC explained by BAC. Only BAC contributed uniquely to the prediction of CC, $t(84)=-4.08, p=.0001$. The model did not approach significance for first run NPE, $F(4,81)=1.15, p=.34$.

\section{BAC Limb}

To investigate the possible biphasic effects of acute alcohol intoxication on second run WCST performance (CC, PE and NPE), a between-groups MANCOVA was performed. All MANCOVA assumptions were deemed to be satisfactory. The Box's M test was non-significant $(p=.41)$ and the Levene's test of equality of error variance across groups was not violated for any of the outcome measures (all $p>.40$ ). The independent variable was position on the BAC curve (ascending or descending) and the covariates were BIS-11, AUDIT and FrSBe scores. There were 22 participants whose BAC levels were either 0 or plateaued during testing; these data were necessarily discarded for this analysis, leaving 33 on the ascending limb and 31 on the descending limb when performing the WCST second run. BAC averaged an identical $.07 \%$ in both groups. The dependent variables were second run CC, PE and NPE. Pillai's trace indicated a non-significant main effect of position on the BAC curve, $F(3,57)=1.27, p=.295$, observed power $=.32$. Participants tested on the ascending limb of the BAC curve did not have significantly different CC scores $(M=2.67, S D=1.21)$ from those tested on the descending limb $(M=2.81, S D=1.17)$. Participants tested on the ascending limb of the BAC curve did not have significantly different PE scores $(M=10.94, S D=6.07)$ from those tested on the descending $\operatorname{limb}(M=12.39, S D=6.13)$. Likewise, participants tested on the ascending limb of the BAC curve did not have significantly different NPE scores $(M=8.33, \mathrm{SD}=4.96)$ when compared to participants tested on the descending $\operatorname{limb}(M=6.97, S D=4.93)$. The results were unchanged even when only participants with BACs of at least .04\% were examined. 


\section{Discussion}

The primary purpose of the present study was to examine the dose-related effects of acute alcohol consumption on executive cognitive performance in naturalistic settings, using the WCST second run PE measure as an operational index of prefrontal cortical dysfunction following the example of an earlier laboratory study (Lyvers \& Maltzman, 1991). Consistent with predictions, BAC accounted for a significant amount of variance in PE scores, but did not predict variance in NPE scores (the control index of general brain dysfunction). The trait measures BIS-11, AUDIT, and FrSBe did not account for a significant amount of variance in either PE or NPE, although they were significantly positively correlated with each other as found in other recent work (Lyvers, Duff \& Hasking, 2010). A secondary aim was to examine the possible biphasic effect of alcohol on WCST performance; however, in contrast to the findings of Pihl et al. (2003), participants on the descending limb of the BAC curve did not differ from those on the ascending limb on neurocognitive performance in the present study. This difference could be a function of methodological differences between the two studies, which used different measures of executive cognition. Further; although the Pihl et al. laboratory study had a much smaller sample size ( $n=$ 11 and $n=10$ for the ascending and descending limb conditions, respectively), they ensured that testing was initiated in all participants at $.08 \%$ BAC whether BAC was ascending or descending, whereas in the present study the assignment to BAC limb was necessarily made post hoc and averaged $.07 \%$ for both BAC limb groups. Further research is needed before conclusions as to BAC limb related differential effects of alcohol on executive cognitive functioning can be drawn.

The present results supported the premise that acute alcohol intoxication at common inebriating doses preferentially disrupts prefrontal cortical functioning and thereby impairs executive cognitive performance, an effect which is detectable by the dual run WCST procedure across both laboratory and naturalistic (bar) settings. The absence of any nonspecific effect as indexed by NPE in the present study - even at quite high BACs - was perhaps surprising and 
suggests that the effects of a more general cortical depressant action of alcohol on WCST performance may not become significant until BACs are very high. By contrast, selective increase in PE was reported even at relatively low BACs averaging .05\% in Lyvers and Maltzman's (1991) study, and the current findings indicated that second run PE were positively related to BAC across a range of 0 to $.15 \%$ in the naturalistic settings of Australian bars. The dual run WCST paradigm employed in the present study has previously been found to be sensitive to drug effects not only for alcohol in social drinkers (Lyvers \& Maltzman, 1991), but also for nicotine in heavy smokers (Lyvers, Maltzman \& Miyata, 1994), methadone in opioid addicts (Lyvers \& Yakimoff, 2003), and caffeine in light caffeine consumers (Lyvers, Brooks \& Matica, 2004). In those studies, an apparently selective frontal lobe type effect (i.e., influencing PE but not NPE on the second run WCST) was indicated by the results for each drug except caffeine, which showed only a nonspecific effect (i.e., reducing both PE and NPE similarly, a general cognitive enhancing effect). However, in contrast to the present findings where acute alcohol intoxication was associated with increased PE in social drinkers, shortterm abstinence from nicotine or methadone in nicotine or opioid dependent participants, rather than acute intoxication on the respective substances, was associated with increased PE in the corresponding studies cited above. Those findings are consistent with other evidence of differential alteration of prefrontal cortical functioning during early abstinence in addicts, including alcoholics, especially during craving states (e.g., Myrick et al., 2004).

Weissenborn and Duka (2003) interpreted the alcohol-induced increase in perseveration reported by Lyvers and Maltzman (1991) as reflecting an alcohol-induced increase in impulsivity. Perseveration in this context means persisting with responses that were previously rewarded, thus if perseveration can be said to reflect impulsivity it would appear to do so only for the reward sensitivity component of impulsivity (Dawe, Gullo \& Loxton, 2004). Given the role of dopamine in promoting approach behaviors following repeated rewards (Schultz, Tremblay \& Hollerman, 2000; Schulz, 2001), alcohol's dopaminergic agonist actions (Olive \& 
Griffin, 2008) would appear to be a plausible explanation at the neural level of alcohol-induced perseveration. Dopamine has an inhibitory effect in the prefrontal cortex when released by the mesocortical component of the dopamine reward system, effectively taking the prefrontal cortex "off-line" to facilitate more habitual responses according to one interpretation (Arnsten \& Goldman-Rakic, 1998). Thus alcohol-induced increase in perseveration may reflect alcohol's ability to temporarily increase reward sensitivity via enhancement of dopamine activity in the reward system. This interpretation may pertain to certain alcohol-related social phenomena such as the so-called "beer goggles" effect, where acute alcohol intoxication increases the perceived attractiveness of opposite sex models (Lyvers, Cholakians, Puorro \& Sundram, 2010b), as well as the elevated self-reported likelihood of risky behavior at higher BACs in response to highly rewarding stimuli (Lyvers, Cholakians et al., 2010a).

The present findings are important insofar as they demonstrate that the results of a carefully controlled laboratory study can be replicated in naturalistic environments where alcohol is frequently consumed. Although alcohol expectancy was not controlled in the present study, there was no need to do so as alcohol expectancy generally does not influence cognitive performance (Hull \& Bond, 1986) and was previously shown to have no effect on any WCST outcome measure (Lyvers \& Maltzman, 1991). The absence of any relationships of the control variables AUDIT, BIS-11 and FrSBe with the primary outcome measure of interest - WCST second run PE - was expected despite the established links between prefrontal cortex dysfunction and chronic heavy alcohol consumption (Volkow et al., 1994), impulsivity (Chen et al., 2007) and FrSBe scores (Grace \& Malloy, 2001). Although high levels of hazardous and harmful drinking were indicated by AUDIT scores, the present sample consisted of young adults, and alcohol-induced brain damage sufficient to impact executive cognitive performance may not be evident until after decades of alcohol abuse (Lyvers, 2000). Further, the BIS-11 and FrSBe scores were within the range recorded in the normative population, as prospective participants who reported histories of learning disabilities, psychiatric or neurological conditions had been 
excluded from the study. Thus the BIS-11 and FrSBe scores in the present sample were presumably not likely to reflect a range of dysfunction of prefrontal cortex that would influence WCST performance, although the possibility of this had to be controlled nonetheless.

The young age of the present sample means that the present results apply only to young adult social drinkers, somewhat restricting the generalizability of the findings. Another issue of possible concern is the fact that intoxicated participants completed the trait questionnaires under the influence of alcohol, which might conceivably have distorted their responses in some way. Indeed, BAC was significantly correlated with scores on the BIS-11, FrSBe, and AUDIT, suggesting a possible influence of alcohol intoxication on questionnaire responding. However, self-reported alcohol consumption (as measured by AUDIT) was similarly correlated with BIS11 and FrSBe scores in non-intoxicated young adults in other recent research (Lyvers, Duff \& Hasking, 2010), thus to the extent that self-reported alcohol intake reflects actual intake, the present finding that BAC (like AUDIT) was related to BIS-11 and FrSBe makes sense without invoking any influence of acute intoxication on questionnaire scores. In other words, among social drinkers, whether intoxicated or not, those who score higher on BIS-11 and FrSBe tend to also score higher on AUDIT and drink more heavily, thus achieving higher BACs when they drink.

In conclusion, supporting previous laboratory research (Lyvers \& Maltzman, 1991; Peterson et al., 1990; Pihl et al., 2003; Weissenborn \& Duka, 2003), the present investigation demonstrated that the acute self-regulated consumption of alcohol can impair executive cognitive functioning in social drinkers under naturalistic drinking conditions. Differential BAC limb effects on executive cognition were not indicated by the present findings, thus further research using larger samples tested across a range of BAC is needed to determine whether performance of executive neurocognitive tests such as the WCST is differentially affected by BAC limb at time of testing. 


\section{References}

Alcolizer Technology. (2007). Operation manual H1/H2. Cleveland, QLD: Author.

Arnsten, A.F.T., \& Goldman-Rakic, P.S. (1984). Selective prefrontal cortical projections to the region of the locus coeruleus and raphe nuclei in the rhesus monkey. Brain Research, 306, 918.

Australian Institute of Health \& Welfare (AIHW). (2008). 2007 National Drug Strategy Household Survey: First results (Cat. no. PHE 98. Drug Statistics Series number 20). Canberra: AIHW.

Axelrod, B. N. (2002). Are normative data from the 64-card version of the WCST comparable to the full WCST? The Clinical Neuropsychologist, 16, 7-11.

Babor, Babor, T. F., de la Fuente, J. R., Saunders, J., \& Grant, M. (1992). The Alcohol Use Disorders Identification Test: Guidelines for use in primary health care (WHO Publication No. 92.4). Geneva, Switzerland: World Health Organization.

Bailey, W. J. (2005). Drug use in American society (6th ed.). Minneapolis: Burgess.

Barratt, E., \& Patton, J. H. (1983). Impulsivity: Cognitive, behavioral, and psychophysiological correlates. In M. Zuckerman (Eds.), Biological bases of sensation seeking, impulsivity, and anxiety (pp. 77-116). Hillsdale, NJ: Lawrence Erlbaum Associates.

Bergman, H., \& Källmén, H. (2002). Alcohol use among Swedes and a psychometric evaluation on the alcohol use disorders identification test. Alcohol and Alcoholism, 37, 245-251.

Berman, K.F., Ostrem, J.L., Randolph, C., Gold, J., Goldberg, T.E., Coppola, R., Carson, R.E., Herscovitch, P., \& Weinberger, D.R. (1995). Physiological activation of a cortical network during performance of the Wisconsin Card Sorting Test: A positron emission tomography study. Neuropsychologia, 33, 1027-1046. 
Berner, M. M., Kriston, L., Bentele, M., \& Harter, M. (2007). The Alcohol Use Disorders Identification Test for detecting at-risk drinking: A systematic review and metaanalysis. Journal of Studies on Alcohol and Drugs, 68, 461-473.

Bohn, M. J., Babor, T. F., \& Kranzler, H. R. (1995). The Alcohol Use Disorders Identification Test (AUDIT): Validation of a screening instrument for use in medical settings. Journal of Studies on Alcohol, 56, 423-432.

Chen, A.C.H., Porjesz, B., Rangaswamy, M., Kamarajan, C., Tang, Y., Jones, K.A., Chorlian, D.B., Stimus, A.T., \& Begleiter, H. (2007). Reduced frontal lobe activity in subjects with high impulsivity and alcoholism. Alcoholism: Clinical and Experimental Research, 31(1), 156-165.

Chiaravalloti, N. D., \& DeLuca, J. (2003). Assessing the behavioral consequences of multiple sclerosis: An application of Frontal Systems Behavior Scale (FrSBe). Cognitive and Behavioral Neurology, 16, 54-57.

Davis, K. C., George, W. H., Norris, G., Schacht, R. L., Stoner, S. A., Hendershot, C.S., \& Kajumulo, K. L. (2009). Effects of alcohol and blood alcohol concentration limb on sexual risk-taking intentions. Journal of Studies on Alcohol and Drugs, 4, 499-507.

Dawe, S., Gullo, M., J., \& Loxton, N. J. (2004). Reward drive and rash impulsiveness as dimensions of impulsivity: Implications for substance misuse. Addictive Behaviors, 29, 1389-1405.

De Wit, H., Metz, J., Wagner, N., and Cooper, M. (1990). Behavioral and subjective effects of ethanol: Relationship to cerebral metabolism using PET. Alcoholism, 14, 482-489.

Dybek, I., Bischof, G., Grothues, J., Reinhardt, S., Meyer, C., Hapke, U., John, U., Broocks, A., Hohagen, F., \& Rumpf, H. J. (2006). The reliability and validity of the Alcohol Use Disorders Identification Test (AUDIT) in a German general practice population sample. Journal of Studies on Alcohol, 67, 473-481.

Finnigan, F., \& Hammersley, R. (1992). The effects of alcohol on performance. In A. P. 
Smith \& D. M. Jones (Eds.), Handbook of human performance, Vol. 2: Health and performance. London: Academic Press.

Fleming, M.F., Mihic, S.J., \& Harris, R.A. (2001). Ethanol. In J.G. Hardman \& L.E. Limbird (Eds.), Goodman \& Gilman's the pharmacological basis of therapeutics $\left(10^{\text {th }}\right.$ ed., pp 429-445). New York: McGraw-Hill.

Giancola, P. R. (2007). The underlying role of aggressivity in the relation between executive functioning and alcohol consumption. Addictive Behaviors, 32, 765-783.

Giancola, P. R., \& Zeichner, A. (1997). The biphasic effects of alcohol on human physical aggression. Journal of Abnormal Psychology, 106, 598-607.

Grace, J. \& Malloy, P. F. (2001). Frontal Systems Behaviour Scale (manual). Lutz, FL: Psychological Assessment Resources.

Grace, J., Stout, J. C., \& Malloy, P. F. (1999). Assessing frontal lobe behavioural syndromes with the frontal lobe personality scale, Assessment, 6, 269-284.

Heaton, R. K. (1981). A manual for the Wisconsin Card Sorting Test. Odessa, FL: Psychological Assessment Resources.

Heaton, R. K., Chelune, G. L., Talley, J. L., Kay, G. G., \& Curtis, G. (1993). Wisconsin Card Sort Test (WCST) manual revised and expanded. Odessa, FL: Psychological Assessment Resources.

Hoaken, P.N.S., Assaad, J.M., \& Pihl, R.O. (1998). Cognitive functioning and the inhibition of alcohol-induced aggression. Journal of Studies on Alcohol, 59, 599-607.

Holdstock, L., \& de Wit, H. (1998). Individual differences in the biphasic effects of ethanol. Alcoholism: Clinical and Experimental Research, 22(9), 1903-1911.

Hull, J.G., \& Bond, C.F. (1986). Social and behavioral consequences of alcohol consumption and expectancy: A meta-analysis. Psychological Bulletin, 99, 347-360.

Hurst, P. M., \& Bagley, S. K. (1972). Acute adaptation to the effects of alcohol. Quarterly Journal of Studies on Alcohol, 33, 358-378. 
Jones, B. M. (1973). Memory impairment on the ascending and descending limbs of the blood alcohol curve. Journal of Abnormal Psychology, 82(1), 24-32.

Kongs, S. K., Thompson, L. L., Iverson, G. L., \& Heaton, R. K. (2000). Wisconsin Card Sorting Test-64 card computerized version. Odessa, FL: Psychological Assessment Resources.

Luengo, M. A., Carrillo-de-la-Pena, M. T., \& Otero, J. M. (1991). The components of impulsiveness: A comparison of the Impulsiveness Questionnaire and the Barratt Impulsiveness Scale. Personality and Individual Differences, 12, 657-667.

Lyvers, M. (2000). Loss of control in alcoholism and drug addiction: A neuroscientific interpretation. Experimental and Clinical Psychopharmacology, 8(2), 225-249.

Lyvers, M., Brooks, J., \& Matica, D. (2004). Effects of caffeine on cognitive and autonomic measures in heavy and light caffeine users. Australian Journal of Psychology, 56, 33-41.

Lyvers, M., Cholakians, E., Puorro, M., \& Sundram, S. (2010a). Alcohol intoxication and self-reported risky sexual behavior intentions with highly attractive strangers in naturalistic settings. Journal of Substance Use, in press.

Lyvers, M., Cholakians, E., Puorro, M., \& Sundram, S. (2010b). Beer goggles: Blood alcohol concentration in relation to attractiveness ratings for unfamiliar opposite sex faces in naturalistic settings. Journal of Social Psychology, in press.

Lyvers, M., Czerczyk, C., Follent, A., \& Lodge, P. (2009). Disinhibition and reward sensitivity in relation to alcohol consumption by university students. Addiction Research \& Theory, 17(6), 668-677.

Lyvers, M., Duff, H., \& Hasking, P. (2010). Risky alcohol use and age at onset of regular alcohol consumption in relation to frontal lobe indices, reward sensitivity and rash impulsiveness. Addiction Research \& Theory, in press.

Lyvers, M. F., \& Maltzman, I. (1991). Selective effects of alcohol on Wisconsin card sorting test performance. British Journal of Addiction, 86, 399-407. 
Lyvers, M., Maltzman, I., \& Miyata, Y. (1994). Effects of cigarette smoking and smoking deprivation on Wisconsin Card Sorting Test performance. Experimental and Clinical Psychopharmacology, 2, 283-289.

Lyvers, M., \& Yakimoff, M. (2003). Neuropsychological correlates of opioid dependence and withdrawal. Addictive Behaviors, 28, 605-611.

Mountain, M.A., \& Snow, W.G. (1993). Wisconsin Card Sorting Test as a measure of frontal pathology: A review. Clinical Neuropsychologist, 7, 108-118.

Mueller, S. T. (2008). PEBL: The psychology experiment building language (Version 0.09) [Computer experiment programming language]. Retrieved January 19, 2009, from http://pebl.sourceforge.net

Myrick, H., Anton, R.F., Li, X., Henderson, S., Drobes, D., Voronin, K., \& George, M.S. (2004). Differential brain activity in alcoholics and social drinkers to alcohol cues: Relationship to craving. Neuropsychopharmacology, 29(2), 393-402.

Norton, L. E., Malloy, P. F., \& Salloway, S. (2001). The impact of behavioural symptoms on activities of daily living in patients with dementia. Journal of Geriatric Psychiatry, 9, $41-48$.

Olive, M.F., \& Griffin, W.C. (2008). Ethanol increases extracellular glutamate levels in the ventral tegmental area via D1 receptor activation. Presented at the Annual Scientific Meeting of the College on Problems of Drug Dependence, June 14-19, San Juan Puerto Rico.

Oscar- Berman, M., \& Marinkovic, K. (2007). Alcohol: Effects on neurobehavioral functions and the brain. Neuropsychological Review, 17, 239-257.

Pal, H. R., Jena, R., \& Yadav, D. (2004). Validation of the Alcohol Use Disorders Identification Test (AUDIT) in urban community outreach and de-addiction center samples in north India. Journal of Studies on Alcohol, 65, 794-800.

Pardo, Y., Aguilar, R. L., Molinuevo, B., \& Torrubia, R. (2007). Alcohol use as a behavioural sign 
of disinhibition: Evidence from J. A. Gray's model of personality. Addictive Behaviors, 32(10), 2398-2403.

Patton, J. H., Stanford, M. S., \& Barratt, E. S. (1995). Factor structure of the Barratt Impulsiveness Scale. Journal of Clinical Psychology, 51, 768-774.

Peterson, J.B., Finn, P.R., \& Pihl, R.O. (1992). Cognitive dysfunction and the inherited predisposition to alcoholism. Journal of Studies on Alcohol, 53, 154-160.

Peterson, J. B., Rothfleisch, J., Zelazo, P. D., \& Pihl, R. O. (1990). Acute alcohol intoxication and cognitive functioning. Journal of Studies on Alcohol, 51(2), 114-122.

Pihl, R. O., Paylan, S. S., Gentes-Hawn, A., \& Hoaken, P. N. S. (2003). Alcohol affects executive cognitive functioning differentially on the ascending versus descending limb of the blood alcohol concentration curve. Alcoholism: Clinical and Experimental Research, 27, 773-779.

Podell, K., Lovell, M., Zimmerman, M., \& Goldberg, E. (1995). The cognitive bias task and lateralized frontal lobe functions in males. Journal of Neuropsychiatry \& Clinical Neurosciences, 7, 491-501.

Reinert, D. F., \& Allen, J. P. (2007). The Alcohol Use Disorders Identification Test: An update on research findings. Alcoholism: Clinical and Experimental Research, 31, $185-199$.

Rubin, A., Migneault, J. P., Marks, L., Goldstein, E., Ludena, K., \& Friedman, R. H. (2006). Automated telephone screening for problem drinking. Journal of Studies on Alcohol, $67,454-57$.

Schultz, W., Tremblay, L., \& Hollerman, J. R. (2000). Reward processing in primate orbitofrontal cortex and basal ganglia. Cerebral Cortex, 10, 272-284.

Schulz, W. (2001). Reward signalling by dopamine neurons. Neuroscientist, 7, 293-302.

Smith, G., Perdices, M., O’Sullivan, B., Large, M., \& Barrett, N. (1997). PET study of the Wisconsin Card Sorting Test and brain activation correlates of repeated testing. Australian 
\& New Zealand Journal of Psychiatry, 31 (Supp.), A39.

Spinella, M. (2004). Neurobehavioral correlates of impulsivity. International Journal of Neuroscience, 114, 95-104.

Spinella, M. (2007). Normative data and a short form of the Barratt impulsiveness scale. International Journal of Neuroscience, 117, 359-368.

Stout, J. C., Ready, R. E., Grace, J., Malloy, P. F., \& Paulsen, J. S. (2003). Factor analysis of the frontal systems behavior scale (FrSBe), Assessment, 10, 79-85.

Stout, J. C., Wyman, M. F., Johnson, S. A., Peavy, G. M., Salmon, D. P. (2003). Frontal behavioural syndromes and functional status in probable Alzheimer disease. American Journal of Geriatric Psychiatry, 11, 683-686.

Strauss, E., Sherman, E. M. S., \& Spreen, O. (2006). A Compendium of neuropsychological tests: Administration, norms, and commentary (3rd ed.). NY: Oxford University Press.

Stuss, D.T., Benson, D.F., Kaplan, E.F., Weir, W.S., Naeser, M.A., Lieberman, I., \& Ferrill, D. (1983). The involvement of orbitofrontal cerebrum in cognitive tasks. Neuropsychologia, $21,235-248$.

Stuss, D. T., Levine, B., Alexander, M. P., Hong, J., Palumbo, C., Hamer, L., Murphy, K. J., \& Izukawa, D. (2000). Wisconsin Card Sorting Test performance in patients with focal frontal and posterior brain damage: Effects of lesion location and test structure on separable cognitive processes. Neuropsychologia, 38(4), 388-402.

Tabachnick, B. G., \& Fidell, L. S. (2007). Using multivariate statistics (5 ${ }^{\text {th }}$ ed.). Boston: Pearson/Allyn \& Bacon.

Tien, A. Y., Spevack, T. V., Jones, D. W., Pearlson, G. D., Schlaepfer, T. E., \& Strauss, M. E. (1996). Computerized Wisconsin Card Sorting Test: Comparison with manual administration. Kaohsiung Journal of Medical Sciences, 12, 479-485.

Weissenborn, R., \& Duka, T. (2003). Acute alcohol effects on cognitive function in social drinkers: Their relationship to drinking habits. Psychopharmacology, 165, 306-312. 
Velligan, D. I., Ritch, J. L., Sui, D., Di Cocco, M., \& Huntzinger, C. D. (2002). Frontal systems behavior scale in schizophrenia: Relationships with psychiatric symptomatology and adaptive function. Psychiatry Research, 113, 227-236. 
Table 1.

Means and standard deviations for BIS-11, AUDIT, FrSBe, BAC, and second run WCST Categories Completed, Perseverative Errors and Non-Perseverative Errors across the entire sample.

\begin{tabular}{lll} 
Variables & $M$ & $S D$ \\
\hline BIS-11 & 67.45 & 11.18 \\
AUDIT & 13.72 & 7.69 \\
FrSBe & 95.88 & 19.65 \\
BAC & $0.05 \%$ & $0.04 \%$ \\
Categories Completed & 2.92 & 1.21 \\
Perseverative Errors & 10.79 & 5.81 \\
Non-Perseverative Errors & 7.00 & 4.67 \\
\hline
\end{tabular}




\section{Table 2.}

Correlations among values for mean Blood Alcohol Concentration (BAC), second run WCST Categories Completed (CC), Perseverative Errors (PE) and Non-Perseverative Errors (NPE), Barratt Impulsivity Scale Version 11 (BIS-11), Alcohol Use Disorders Identification Test (AUDIT), Frontal Systems Behavior Scale (FrSBe), and Age.

\begin{tabular}{|c|c|c|c|c|c|c|c|c|}
\hline Variables & 1 & 2 & 3 & 4 & 5 & 6 & 7 & 8 \\
\hline 1. BAC & -- & $-.29 * *$ & $.34 * *$ & .19 & $.28 * *$ & $.35 * *$ & $.24^{*}$ & $.23 *$ \\
\hline 2. $\mathrm{CC}$ & & -- & $-.63 * *$ & $-.65 * *$ & -.13 & -.04 & -.05 & .02 \\
\hline 3. PE & & & -- & $.24 *$ & .11 & .10 & -.01 & .18 \\
\hline 4. NPE & & & & -- & .12 & -.00 & .05 & -.01 \\
\hline 5. BIS-11 & & & & & -- & $.41 * *$ & $.56 * *$ & .11 \\
\hline 6. AUDIT & & & & & & -- & $.47 * *$ & -.03 \\
\hline 7. FrSBe & & & & & & & -- & -.20 \\
\hline 8. Age & & & & & & & & -- \\
\hline
\end{tabular}

$* p<.05 * * p<.01$ 
Table 3.

Regression analysis predicting second run WCST perseverative errors.

\begin{tabular}{lccccccc}
\hline & $\mathrm{B}$ & $\beta$ & $\mathrm{R}$ & $\mathrm{R}^{2}$ & $\Delta \mathrm{R}^{2}$ & $\mathrm{df}$ & $\mathrm{F}$ \\
\hline Step 1 & & & .166 & .027 & & 82 & .770 \\
BIS-11 & .076 & .147 & & & & & \\
AUDIT & .076 & .101 & & & & & \\
FrSBe & -.041 & -.140 & & & & & \\
\hline Step 2 & & & .364 & .132 & .105 & 81 & $3.084^{*}$ \\
BAC & 50.23 & $.351^{* *}$ & & & & & \\
& & & & & & & \\
\hline
\end{tabular}

$* \mathrm{p}<.05, * * \mathrm{p}<.01$ 
Table 4.

Regression analysis predicting second run WCST non-perseverative errors.

\begin{tabular}{lccccccc}
\hline & $\mathrm{B}$ & $\beta$ & $\mathrm{R}$ & $\mathrm{R}^{2}$ & $\Delta \mathrm{R}^{2}$ & $\mathrm{df}$ & $\mathrm{F}$ \\
\hline Step 1 & & & .132 & .017 & & 82 & .482 \\
BIS-11 & .060 & .145 & & & & \\
AUDIT & -.038 & .077 & & & & & \\
FrSBe & .000 & -.001 & & & & & \\
\hline Step 2 & & & .230 & .053 & .035 & 81 \\
BAC & 23.49 & .204 & & & & & \\
& & & & & & & \\
\end{tabular}


Figure 1. Perseverative errors on the second run of the Wisconsin Card Sorting Test (PE2) as a function of BAC averaged over three measurements during testing (MEANBAC).

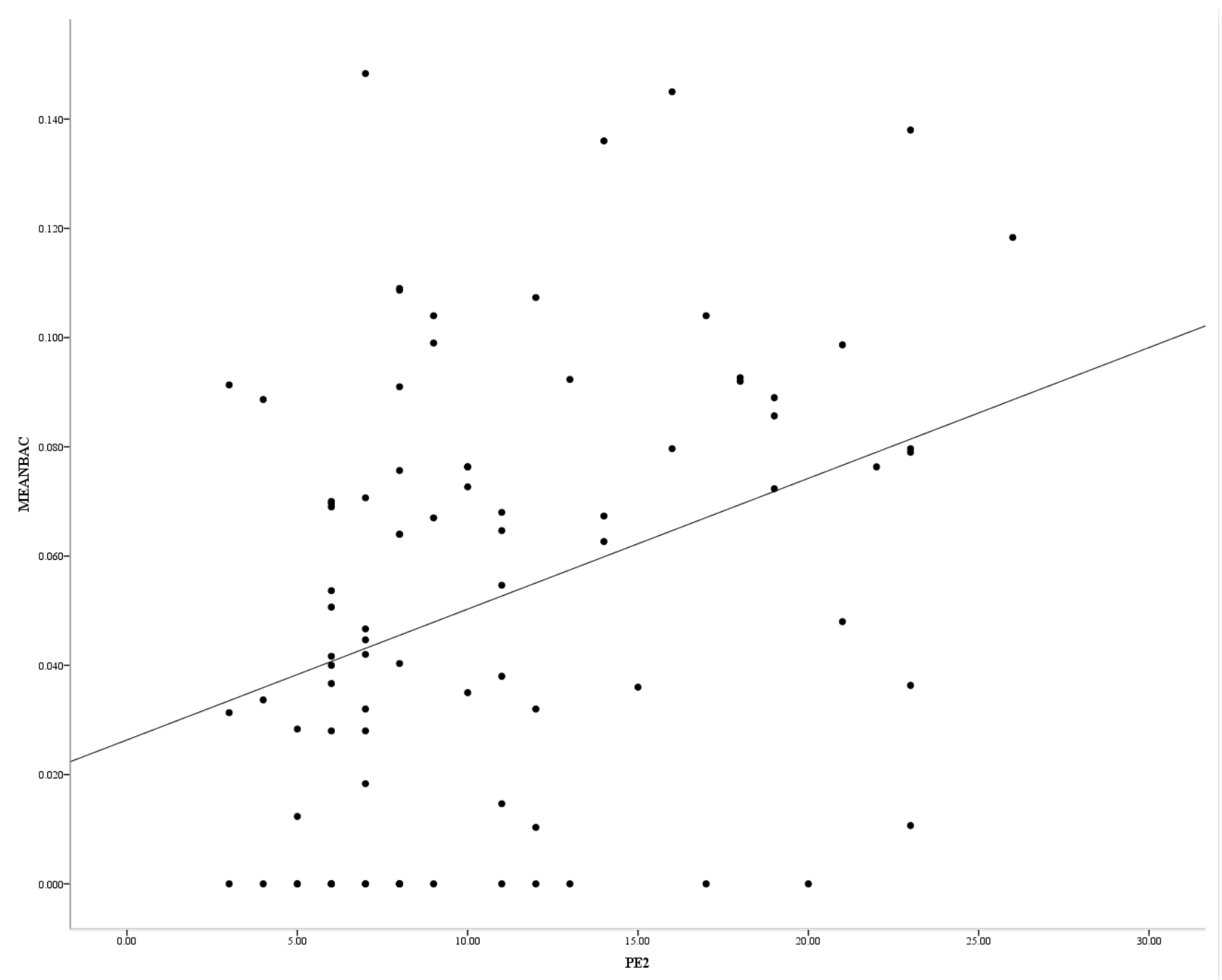

